\title{
NuSTAR observatory science operations: on-orbit acclimation
}

\author{
Karl Forster*a , Fiona A. Harrison ${ }^{\mathrm{a}}$, Suzanne R. Dodd ${ }^{\mathrm{b}}$, Daniel K. Stern ${ }^{\mathrm{b}}$, Hiromasa Miyasaka ${ }^{\mathrm{a}}$, \\ Kristin K. Madsen ${ }^{\mathrm{a}}$, Brian W. Grefenstette ${ }^{\mathrm{a}}$, Craig B. Markwardt ${ }^{\mathrm{d}}$, William W. Craig ${ }^{\mathrm{c}}$, Francis E. \\ Marshall $^{\mathrm{d}}$ \\ ${ }^{\mathrm{a}}$ Cahill Center for Astronomy and Astrophysics, California Institute of Technology, $1200 \mathrm{E}$. \\ California Blvd., Pasadena, CA 91125; ' Jet Propulsion Laboratory, 4800 Oak Grove Dr, Pasadena, \\ CA 91109; 'Space Sciences Laboratory, University of California, Berkeley, 7 Gauss Way, Berkeley, \\ CA 94720; ${ }^{\mathrm{d} N A S A}$ Goddard Space Flight Center, Greenbelt, MD.
}

\begin{abstract}
The Nuclear Spectroscopic Telescope Array (NuSTAR) is the first focusing high energy (3-79 keV) X-ray observatory. The NuSTAR project is led by Caltech, which hosts the Science Operations Center (SOC), with mission operations managed by UCB Space Sciences Laboratory. We present an overview of NuSTAR science operations and describe the on-orbit performance of the observatory. The SOC is enhancing science operations to serve the community with a guest observing program beginning in 2015. We present some of the challenges and approaches taken by the SOC to operating a full service space observatory that maximizes the scientific return from the mission.
\end{abstract}

Keywords: NuSTAR, NASA small explorer, X-ray optics, CZT detectors, Science Operations, Metrology

\section{INTRODUCTION}

Development of operations systems and processes for low cost space observatories must be cognizant of the paradigm of decreasing operations costs during extended mission phases. Space observatories are a finite resource where the preliminary objective of mission operations is to support a short primary mission (usually 2-5 years) with no guarantee of funds being available for extensions past the original contract. The primary operations goal is always to optimize scientific output of each observatory within a finite lifetime and with limited and decreasing funds. We present an overview of the science operations for the NASA's latest space telescope and describe the adjustments that were needed to respond to the on-orbit performance of the observatory.

The Nuclear Spectroscopic Telescope Array $\left(\mathrm{NuSTAR}^{1}\right.$ is the newest space astrophysics observatory within NASAs small explorer program. These missions are constrained to be low cost with a short development and construction phase, and designed to achieve specific scientific goals, often a consequence of technological advances in telescope and/or detector capabilities. Specific advances in lightweight X-ray optics and mirror coatings and the development of high efficiency solid state CdTeZn detectors were major elements in the selection of NuSTAR, the first high energy ( $3-79$ $\mathrm{keV}$ ) X-ray focusing telescope in orbit.

\subsection{The NuSTAR observatory}

The NuSTAR observatory consists of two co-aligned hard X-ray telescopes pointed at celestial targets by a three axis stabilized spacecraft based on Orbital Science's LeoStar-2 bus. Spacecraft attitude is determined using three star cameras $^{2}$ mounted on the spacecraft bus. The X-ray optics and detector benches are separated by a stiff mast that includes a mechanism with the capability of making small adjustments to optimize the location of the optical axis on the focal plane detectors (see Fig.1). The X-ray optics bench consists of two Wolter-I conical approximation ${ }^{3}$ mirror modules, each with 133 shells coated with multiple layers of W/Si and $\mathrm{Pt} / \mathrm{C}$, limiting the highest efficient reflectivity to the Pt $78.4 \mathrm{keV} \mathrm{K}$-edge ${ }^{4,5,6}$. The focal plane bench is mounted on the spacecraft bus and consists of two independent solid state photon counting detector modules (FPMA \& B), each with a 2x2 array of CdZnTe (CZT) crystal detectors, surrounded by CsI anti-coincidence shielding. The detectors are passively cooled and operate at $15^{\circ} \mathrm{C}^{7}$.

* krl@srl.caltech.edu; phone 1-626-395-2101; nustar.caltech.edu

Observatory Operations: Strategies, Processes, and Systems V, edited by Alison B. Peck,

Chris R. Benn, Robert L. Seaman, Proc. of SPIE Vol. 9149, 91490R - @ 2014 SPIE

CCC code: $0277-786 \mathrm{X} / 14 / \$ 18 \cdot$ doi: $10.1117 / 12.2056916$

Proc. of SPIE Vol. 9149 91490R-1 

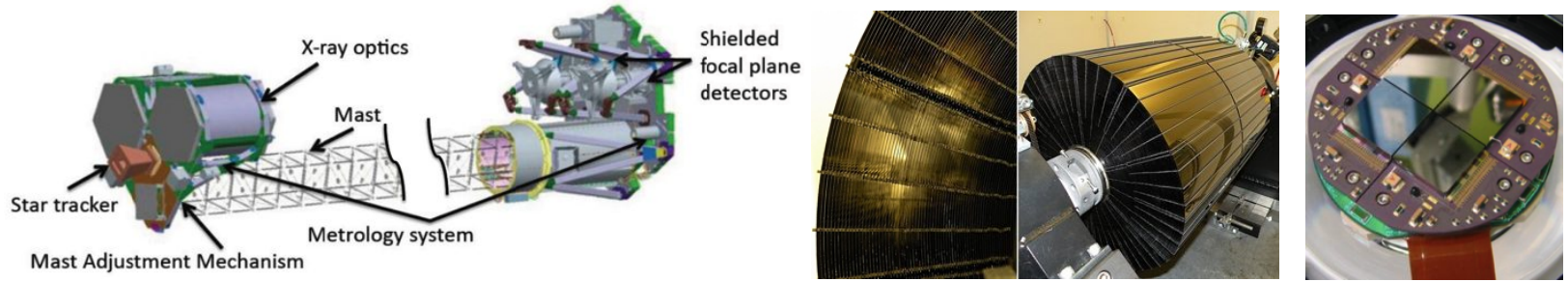

Figure 1. Left - Model of the NuSTAR telescopes. Center - Photographs of one of the two optics modules. Right - One of the two detector modules with 4 CZT detector crystals.

Due to thermal gradients changing as a function of orbit, the mast does not maintain the relative alignment between the optics and focal plane benches. So NuSTAR operates an aspect-metrology system that consists of a star tracking camera mounted on the optics bench, to give absolute pointing, and two laser metrology units which measure the translation, tip, tilt and clocking between the benches (see Fig. 1). This information is used to reconstruct the instantaneous instrument alignment (position of the focal point on the detectors) and the telescope pointing direction. The resulting performance of the observatory is given in Table 1.

Table 1. NuSTAR observatory performance

\begin{tabular}{|c|c|c|c|}
\hline \multicolumn{2}{|l|}{ Spacecraft } & \multicolumn{2}{|l|}{ Telescopes (2) } \\
\hline Launch (Orbital Pegasus) & June 13,2012 & Focal length & $1014 \mathrm{~cm}$ \\
\hline Bus & Orbital LeoStar-2 & Field of view & $12.2 \operatorname{arcmin}$ \\
\hline ACS & 3x DTU Star trackers & Angular resolution & 18'FWHM) \\
\hline Mass / Power & $350 \mathrm{~kg} / 650 \mathrm{~W}$ & Aspect reconstruction (bright source) & $1.5^{\prime \prime}(1 \sigma)$ \\
\hline \multicolumn{2}{|l|}{ Orbit } & \multicolumn{2}{|l|}{ Focal plane CdZnTe detectors (2) } \\
\hline Inclination & 6 degrees & Size & $2 \times 2 \mathrm{~cm}$ \\
\hline Altitude & 610 to $650 \mathrm{~km}$ & Pixels & $160 \times 160$ \\
\hline Period & 97.126 minutes & Pixel pitch & $2.45 \operatorname{arcsec}$ \\
\hline \multirow[t]{2}{*}{ Ground station } & Malindi (ASI), Kenya & Maximum readout rate & 400 events/s \\
\hline & & Energy range & to $78.4 \mathrm{keV}$ \\
\hline \multicolumn{2}{|l|}{ Operations } & Spectral resolution & $400 / 900 \mathrm{eV}$ at $10 / 68 \mathrm{keV}$ \\
\hline \multicolumn{2}{|l|}{ Sun / Full-Moon avoidance* } & Temporal resolution & \multirow[t]{2}{*}{$3 \mathrm{~ms}$} \\
\hline Slew rate & 0.06 degrees / second & \multirow{2}{*}{\multicolumn{2}{|c|}{ *NuSTAR is designed to be able to observe the Sun }} \\
\hline ToO response time & $<24$ hours & & \\
\hline
\end{tabular}

\section{MISSION OPERATIONS}

NuSTAR operations is a collaboration between the Science Operations Center (SOC), in the Cahill center for Astronomy and Astrophysics at the California Institute of Technology (Caltech), Pasadena CA, and the Mission Operations Center (MOC) managed by the UC Berkeley Space Sciences Laboratory (SSL), Berkeley CA. SSL has more than a decade of flight operations experience and currently operates a fleet of seven NASA research satellites. This provides the NuSTAR mission with highly automated and extremely cost-effective operations, such that mission operations as a fraction of the overall NuSTAR budget is lower then average for astrophysics explorer missions. Details of NuSTAR on-orbit mission operations are presented in a companion paper in this volume ${ }^{8}$.

The NuSTAR SOC performs all science planning and data processing functions and monitors the health of the instrument and telescope. This includes survey and observation planning, observatory scheduling, and monitoring survey progress to maximize the scientific return from the mission. Automated data processing is performed daily at the SOC and includes monitoring the data quality and the validation of science processing. The SOC leads observatory calibration activities, including ground based and in-orbit measurements, and delivers calibrated data products to the NuSTAR science team and the public through the High Energy Astrophysics Archive (HEASARC) at the Goddard Space Flight Center. Data processing software has been developed at the SOC in collaboration with software engineers at the ASI Science Data Center (ASDC, Italy). This software is available to the public, as are on-line tools for observation constraint checking that are useful for the community in the observation proposal process. 


\subsection{MOC - SOC interface}

Communication between the MOC and SOC is primarily via a two-way electronic interface that allows the SOC to transmit telescope pointing requests and instrument configuration information, which are automatically validated and constraint checked by the MOC, and to receive raw data products from the MOC that are automatically generated after every ground station contact. The SOC has access to telemetry trending analysis tools and web-based monitoring of the observatory hosted at the MOC. An automated anomaly paging system alerts the MOC and SOC teams to any issues detected during contacts ${ }^{8}$.

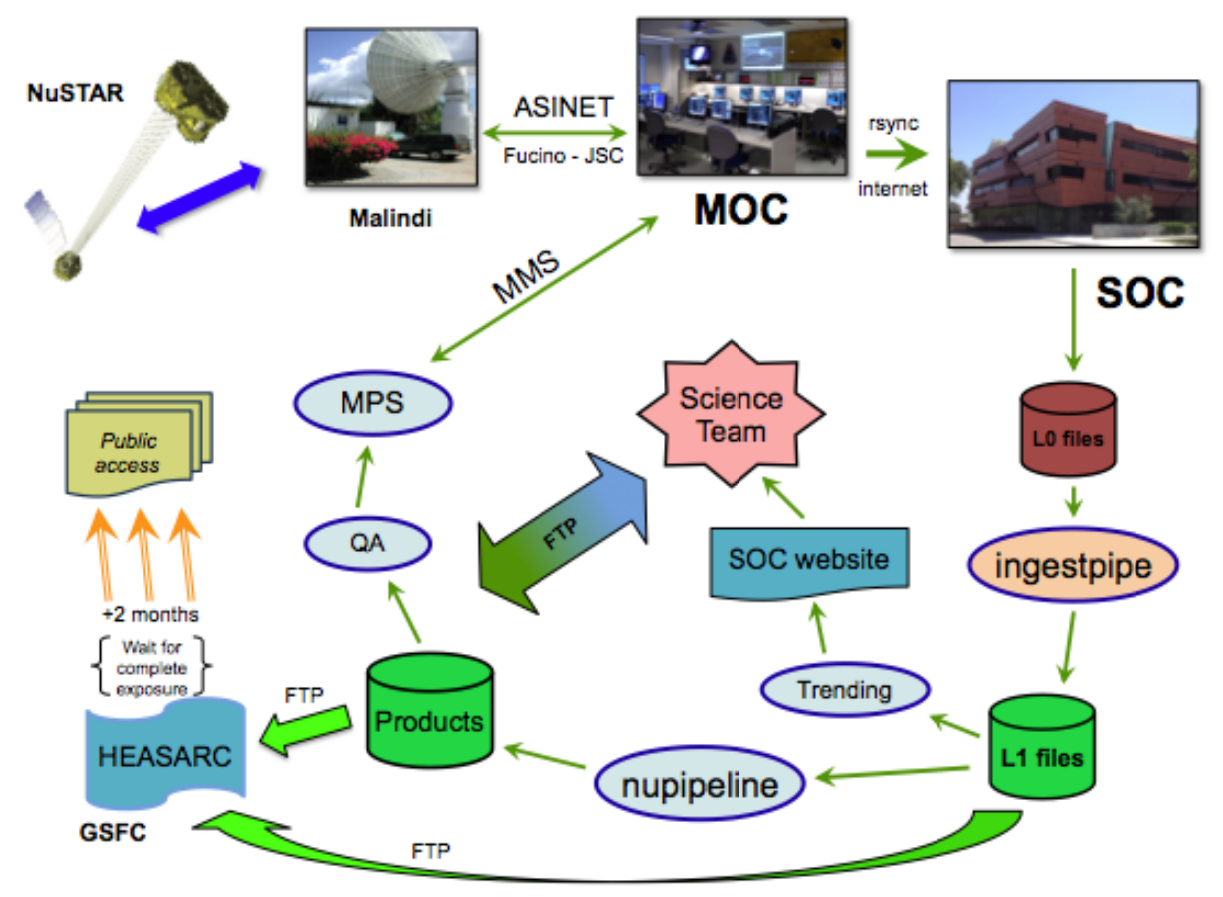

Figure 2. Schematic of NuSTAR science operations. Automated data processing at the SOC takes raw (L0) files obtained from the MOC and generates calibrated science products for delivery to the public archive at HEASARC. Results from the SOC QA system inform science planners and feed back into the mission planning system (MPS).

Observatory pointing requests are transmitted from the SOC to the MOC via a messaging system (MMS), developed at SSL based on a Subversion $\left(\mathrm{SVN}^{9}\right)$ revision control system, which is hosted at SSL with clients at the SOC and MOC. An Observatory Pointing Request Message (OPRM) is delivered to the MMS via a "commit" operation at the SOC. The SVN client then transmits the file to the message system repository at the MOC. An SVN hook script at the MOC automatically performs software validations on the pointing requests contained within the OPRM. If a message is rejected for any reason, the MOC and SOC teams are notified by the MMS; adjustments are then made at the SOC, and a new version of the OPRM is committed.

Delivery of an OPRM is performed once per week and each OPRM contains information for all the observations scheduled in a 9-day period starting on Mondays at 0000 UTC. During nominal operations an OPRM is transmitted during office hours the week prior to the period covered by the OPRM. Each pointing request within the OPRM (called a "block") contains the time of the start of a slew to a new target, the target name and coordinates, as well as the expected instrument count rate for the observation. The MOC performs a weekly upload of a command list in an absolute time sequence (ATS) file that includes station contact commanding as well as observatory slew commands based on the OPRM. The spacecraft autonomously executes these commands at the time associated with the command in the ATS. A new ATS is uploaded and activated before the end of a currently running ATS.

NuSTAR science data and engineering housekeeping are processed automatically at the SOC upon receipt of raw data files from the MOC, typically about four times every day and within an hour of the end of each ground station contact. 


\subsection{Ground station support}

Ground station support scheduling is based on the expected data volume for the targets tabulated in each OPRM block. Standard operations support uses four contacts per day through the Italian Space Agency's (ASI) Malindi ground station in Kenya. This provides approximately $100 \%$ margin for data recovery during nominal science operations. Additional ground station passes are scheduled to support the higher data volume observations of bright X-ray sources, like the Crab Nebula, using Kongsberg Satellite Services antenna in Singapore and Universal Space Network's antennas at South Point, Hawaii. Data recovery during the primary mission as been outstanding, with $>99.995 \%$ of all science data downlinked and delivered to the SOC. This is in large part due to the automation of ground station contact activities at the MOC, including software that detects data transmission dropouts and autonomously sends retransmission commands to the spacecraft. This has resulted in only 2 seconds of science data lost in the last year of operations ${ }^{8}$.

\section{OBSERVATION PLANNING}

NuSTAR was designed to be able to access $80 \%$ of the sky at any time and to stare at a location on the celestial sphere for periods as long as a month with a duty cycle of $>50 \%$. This observatory efficiency is primarily determined by the occultation of a target by the Earth during each orbit. The detectors remain active during Earth occultation, essentially recording instrumental background. Each observation thus consists of an occulted and un-occulted period each orbit with exposure time built up over multiple orbits in $\sim 3000$ second exposures. The duration of each un-occulted period depends on the plane of the NuSTAR orbit at the time of the observation. The NuSTAR orbit inclination is $6^{\circ}$ so for targets with low Declinations $\left(|\mathrm{Dec}|<55^{\circ}\right)$ there is at most a $15 \%$ range in un-occulted duration over the $\sim 50$-day nodal precession period. Targets at higher Declinations have a larger variation of available exposure time and for observations with exposure time goals that will take longer then one day to achieve, these higher efficiency dates are taken into account during target scheduling.

Other considerations for scheduling observations include:

- Passage of the spacecraft through the South Atlantic Anomaly (SAA) where the higher level of particle induced background is detected by the anti-coincidence shielding. During these passages the CZT detectors remain active but the X-ray events are not recorded because the increased background would swamp the signal from astrophysical sources (and increase onboard data accumulation). SAA passages occur in approximately $50 \%$ of all orbits and can last up to 850 seconds. The average impact of SAA passages on the available exposure time for an observation is $<10 \%$.

- NuSTAR spacecraft attitude is determined from three star tracker cameras mounted on the spacecraft bus (called Camera Head Units, or CHU1,2,3) and at least one of these cameras must provide a valid attitude solution at all times. There are periods when an observation would result in all three CHU occulted by the Earth, Moon, or Sun. These CHU blockage scheduling constraints can last up to 3 days.

- The NuSTAR telescope is designed to perform Solar observations so the Sun avoidance angle is determined from the constraints on the absolute pointing determination from the star tracker mounted on the optics bench (CHU4). The baffling around CHU4 enables valid attitude solutions when the Sun is $>39^{\circ}$ from the telescope boresight, but the operational limit for Sun avoidance is $>50^{\circ}$ due to solar array limits.

- Moon avoidance angle is determined from measurements of CHU4 performance during observations close to the full Moon and is set to $>14^{\circ}$.

- Some observations, particularly of extended sources or survey observations with overlapping tiles, will have constraints on the Position Angle (PA) during an observation. A PA requirement for an observation results in a scheduling constraint because the power and thermal limits for the spacecraft determine the roll angle of the telescope boresight to the Sun-Earth vector. There is a $10^{\circ}$ margin allowed for this constraint, and so a particular PA can only be maintained for a limited duration, from 5 days to a few months depending on the ecliptic latitude of a target (see Fig. 3).

The optimal schedule for observations minimizes the slew time between targets and maximizes the available exposure time for each observation. This is parameterized as an "observation efficiency", with a requirement that the average for the entire mission be $>50 \%$, and has consistently remained above $54 \%$ during the primary mission. 

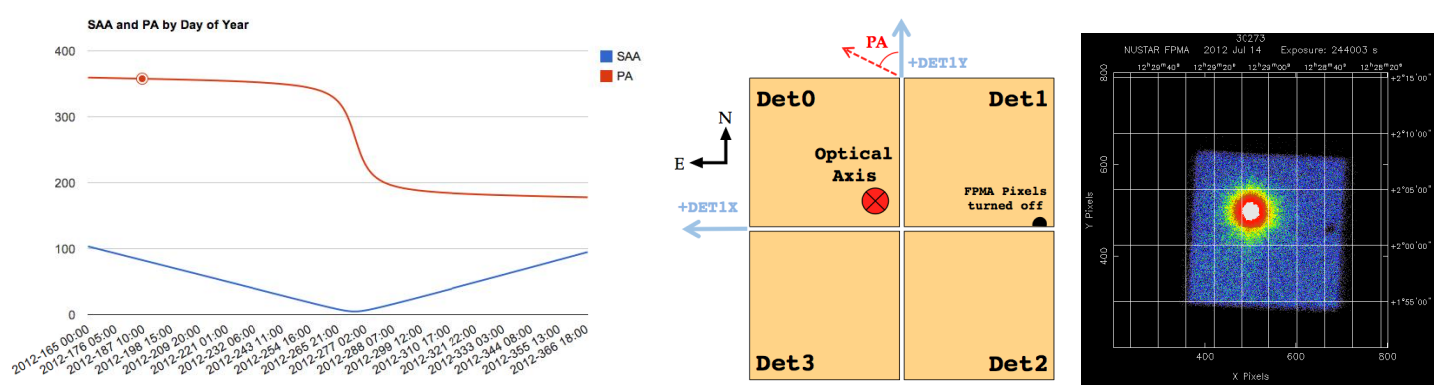

Figure 3. Left - Example of the variation of position angle (red) and Solar aspect angle (blue) for 3C 273 (J2000 $187^{\circ} .278$ $+2^{\circ} .052$ ) during June-Dec 2012. Center - Definition of NuSTAR PA and the nominal position of the telescope optical axis approximately $3 \mathrm{~mm}\left(60^{\prime \prime}\right)$ from the center of the field of view, on DET0. Right - Image from the observation of 3C273 on 2012-07-14 at $\mathrm{PA}=355^{\circ}(\mathrm{ObsID}=10002020001)$.

\subsection{Monthly MPS updates}

The predicted time of Earth occultation for each target in the planned target database is updated monthly based on the latest measurement of the spacecraft orbital Two Line Elements (TLE), provided by the MOC, and are generally accurate to $\pm 10 \mathrm{~s}$ for up to 60 days in the future. A similar update is performed monthly for SAA passage times. These are combined with Sun, Moon, and CHU blockage constraints to generate predicted efficiency data for each target in the target database. The optimal time for an observation to be scheduled is usually within 5 days of peak efficiency.

An example of the variation in observation efficiency is shown in Fig. 4 below for 3C 273 and NGC 3516.
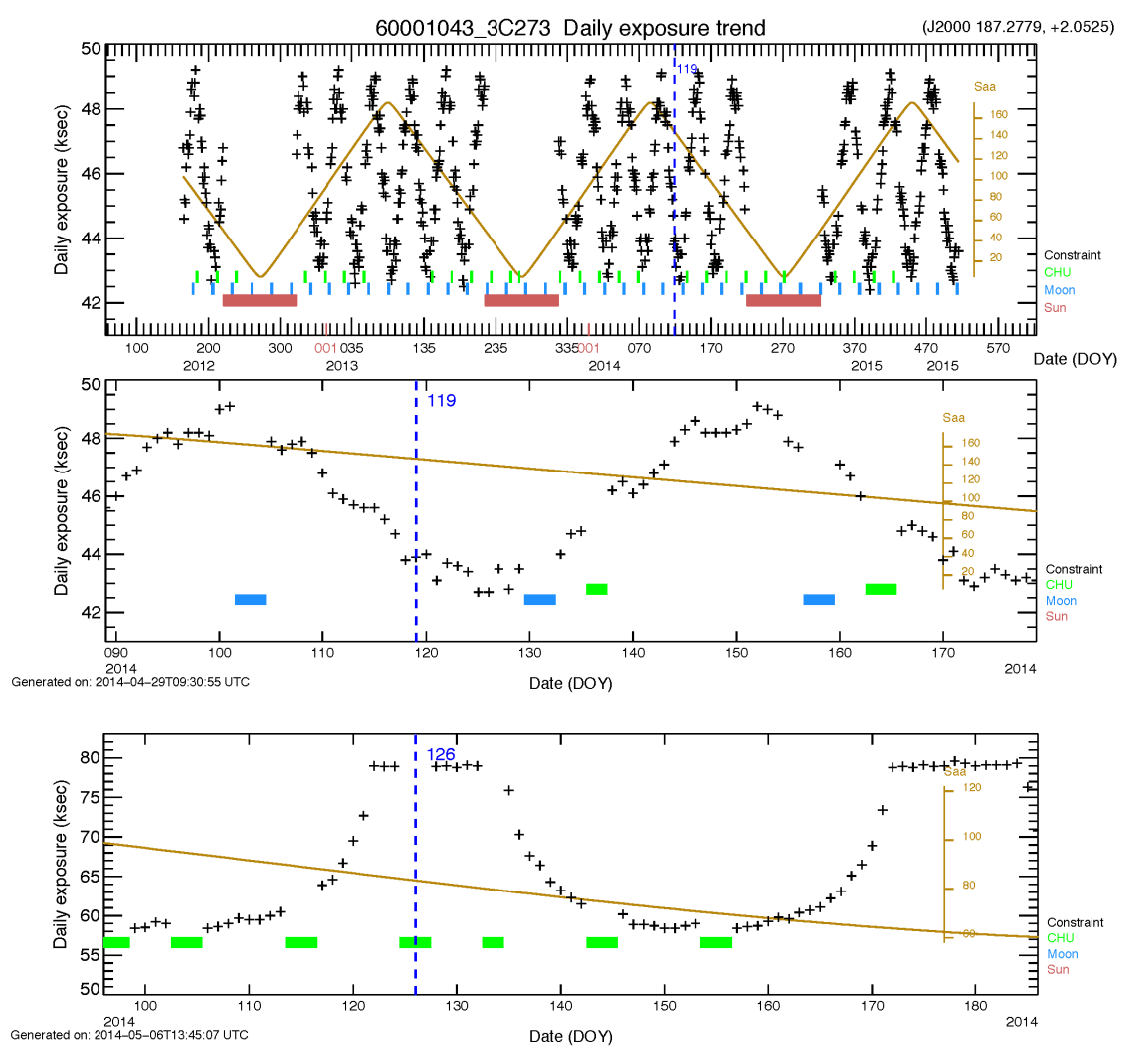

Figure 4. Observation efficiency plots for two targets of different Declinations. Top - Modulation of available daily exposure time for 3C 273 (Dec $+2^{\circ} .05$ ) from June 2012 to May 2015. The Solar aspect angle each day is plotted in yellow. Center - Expanded plot near the date of latest TLE (blue dashed line). Lower - Available daily exposure time trend for NGC $3516\left(\operatorname{Dec}+72^{\circ} .57\right)$. 


\subsection{Coordinated observations}

NuSTAR complements observations using current soft X-ray observatories like Chandra, XMM-Newton, Suzaku, and Swift and is a bridge to low resolution non-focusing or coded mask instruments operated in the hard X-ray band by INTEGRAL, Fermi, MAXI, and Swift-BAT. The capabilities of NuSTAR are being used to break model degeneracy within interpretations from soft X-ray observations alone. This capability was understood before launch and a collaboration between the NuSTAR and Swift missions has been undertaken where all NuSTAR observations have simultaneous Swift-XRT observations of $2 \mathrm{ksec}$ or more. In addition, more then 1.5 Msec of XMM-Newton observation time, including two large programs, were awarded to the NuSTAR team in AO-11 even before the NuSTAR launch.

Joint observation programs have also been offered to the community within the 2013 proposal calls for XMM-Newton and Chandra observatories with NuSTAR observing time allocated before the implementation of any NuSTAR guest observer program. This is a significant investment in observatory time $(>10 \%)$ during the primary mission, and the interest in exploiting the capability of coordinated observations in the soft and hard X-ray bands is high; the $1.5 \mathrm{Msec}$ of NuSTAR time offered in the latest XMM-Newton AO was oversubscribed by a factor of six.

The level of coordination with other observatories, including ground-based observatories like HESS, MAGIC, and VERITAS, is unprecedented for a new mission; with more then $15 \%$ of all NuSTAR observations requiring some coordination of scheduling. Scheduling constraints for NuSTAR are mild in comparison with other space observatories and so the NuSTAR schedule will most often follow the timing of observations for other observatories. The flexibility in the NuSTAR MPS allows straightforward determination of target visibility and rapid scheduling adjustment.

\subsection{ToO response}

NuSTAR is designed to be able to access $80 \%$ of the sky at any given time and this makes it a powerful telescope for Target of Opportunity (ToO) investigations. The streamlined operations model for NuSTAR, accomplished in large part by the successful automation of operations at the MOC, minimizes operations costs but results in response times to ToOs limited by staffing in the SOC and MOC (standard business hours) and the availability of ground station or TDRSS contacts (4 / day).

It was expected before launch that only a handful of ToO's would be executed during the primary mission, but the community interest is high in exploiting NuSTAR for studies of transient and time-variable phenomena, and on average two ToOs have been executed every month. The majority of these ToOs have required a response time of $<7$ days, but the record for response from $\mathrm{ToO}$ request to the telescope on target is 6.5 hours.

\section{DATA ANALYSIS}

NuSTAR data analysis is performed by a software package called NuSTARDAS developed at the ASI Science Data Center (ASDC, Italy) in collaboration with the NuSTAR SOC. The design of the NuSTARDAS pipeline software and calibration files is based on community standard implementations used in analysis of data from high-energy astrophysics observatories. This reduces the data analysis effort because of the community's familiarity with standard tools and protocols. NuSTARDAS is fully compatible and integrated with the HEASoft software package, maintained and distributed by the NASA-HEASARC data center $^{10}$, and is officially distributed to users within its standard public software releases.

The NuSTARDAS package produces cleaned and calibrated detector event list files and standard high-level scientific products, starting from the FITS formatted telemetry data following the NASA-OGIP standards ${ }^{11}$. The software is designed as a collection of modules (tasks), written in the HEASoft FTOOLS style, each dedicated to a single function. The package also includes a pipeline processing script ('nupipeline') allowing users to automatically run in sequence all the tasks required for data processing.

The NuSTARDAS parameter interface is implemented with the HEASoft Parameter Interface Library and the I/O with the FITS data files makes use of the FITSIO library ${ }^{12}$. NuSTARDAS makes exclusive use of open source software (C and Perl languages) and multi-mission FTOOLS from the HEASoft package are also used in several NuSTARDAS modules. NuSTARDAS tasks retrieve observatory calibration files that are complaint with the standard structures of HEASARC's calibration database (CALDB) system. 


\subsection{Public access of NuSTARDAS}

The NuSTARDAS package has been designed to allow the community to reproduce any stage of the NuSTAR data processing, which could be necessary, for example, because of improved calibration information, or updated software modules. It is also often the case that detailed scientific analysis of observations requires use non-standard data processing settings and this is achieved through the interface of parameters in the nupipeline script and the NuSTARDAS packages.

NUSTARDAS is integrated within the HEASoft data analysis package and can be freely downloaded from HEASARC (http:/heasarc.gsfc.nasa.gov/docs/software/lheasoft/) and is available for multiple operating system platforms. A software users guide with detailed information and processing examples is available on the NuSTAR archive website (http:/heasarc.gsfc.nasa.gov/docs/nustar/index.html). Updates to NuSTARDAS and CALDB are expected throughout the mission as refinements of data processing tools and calibration updates become available.

\section{DATA ARCHIVE}

The adoption of common standards for data formats, calibration, and analysis software by the high-energy astrophysics community began in 1990 with the creation of the NASA's High Energy Astrophysics Science Archive Research Center $\left(\right.$ HEASARC $\left.^{10}\right)$ at the Goddard Space Flight Center in Greenbelt, MD. HEASARC pioneered a model of data centers staffed by research scientists that has been adopted by science archives for infrared (IRSA, Caltech ${ }^{13}$ ) and optical/UV $\left(\right.$ MAST, STSci ${ }^{14}$ ) astronomical data. The data and software standards developed by the HEASARC provide the underlying infrastructure for the curation and interpretation of data from a wide variety of missions, substantially reducing mission costs while increasing science return.

HEASARC is the primary archive for NuSTAR data, calibration files and data analysis software. The HEASARC's archive services allow scientists to identify, display, cross-correlate, download and analyze data from a variety of past and active missions - including ASCA, BeppoSAX, Chandra, CGRO, Einstein, Fermi, INTEGRAL, ROSAT, RXTE, Suzaku, Swift, WMAP and XMM-Newton - and provides access to a wide range of multi-wavelength sky surveys, astronomical catalogs and other resources.

The NuSTAR data archive is also mirrored at the Agenzia Spaziale Italiana (ASI) Science Data Center (ASDC, Rome) that supports the multi-mission archive for European space astrophysics.

\section{OBSERVATORY ON-ORBIT PERFORMANCE}

The performance of the observatory has been verified with extensive ground calibration and modeling performed prior to launch, refined with a number of calibration observations and activities during a one month in-orbit checkout period after launch, but also utilizing science observations from the first two years of operations ${ }^{15}$.

\subsection{Solar activity}

The sensitivity of the NuSTAR instrument to changes induced in the SAA by varying levels of Solar activity is higher then expected from pre-launch modeling and has required development of additional screening criteria in data processing. The screening identifies SAA passages by comparing the high gain instrument shield event rates to increases in CZT detector count rates. Fig. 5 illustrates the stability of background event rates $(<1 \mathrm{cts} / \mathrm{s})$ during an observation with minimal Solar activity. NuSTARDAS parameters saamode = Optimized and Strict may be used to filter increased background periods around the SAA. The example in the middle panel of Fig. 5 shows the spatial effect of the different SAA filter modes for an observation on 2014-05-05 where the geomagnetic activity index rose to $\mathrm{Kp}=4$.

Investigation of the time variation of NuSTAR instrument background rates has also identified a North West spur to the SAA ("The Tentacle"). Removal of these intervals is performed within NuSTARDAS by monitoring the increase in detector background event rates as the spacecraft is entering the SAA. The NuSTARDAS parameter tentacle $=$ yes will filter these periods of higher background, the example in the lower panels of Fig. 5 is from an observation on 2013-1008 when the geomagnetic activity index rose to $\mathrm{K}_{\mathrm{p}}=5$ (NOAA space weather geomagnetic storm level G1). The algorithm for these SAA related filters has been verified for observations of steady sources with fluxes not dominating 
over the detector background. This screening is most useful for NuSTAR survey programs and investigations of faint source variability.
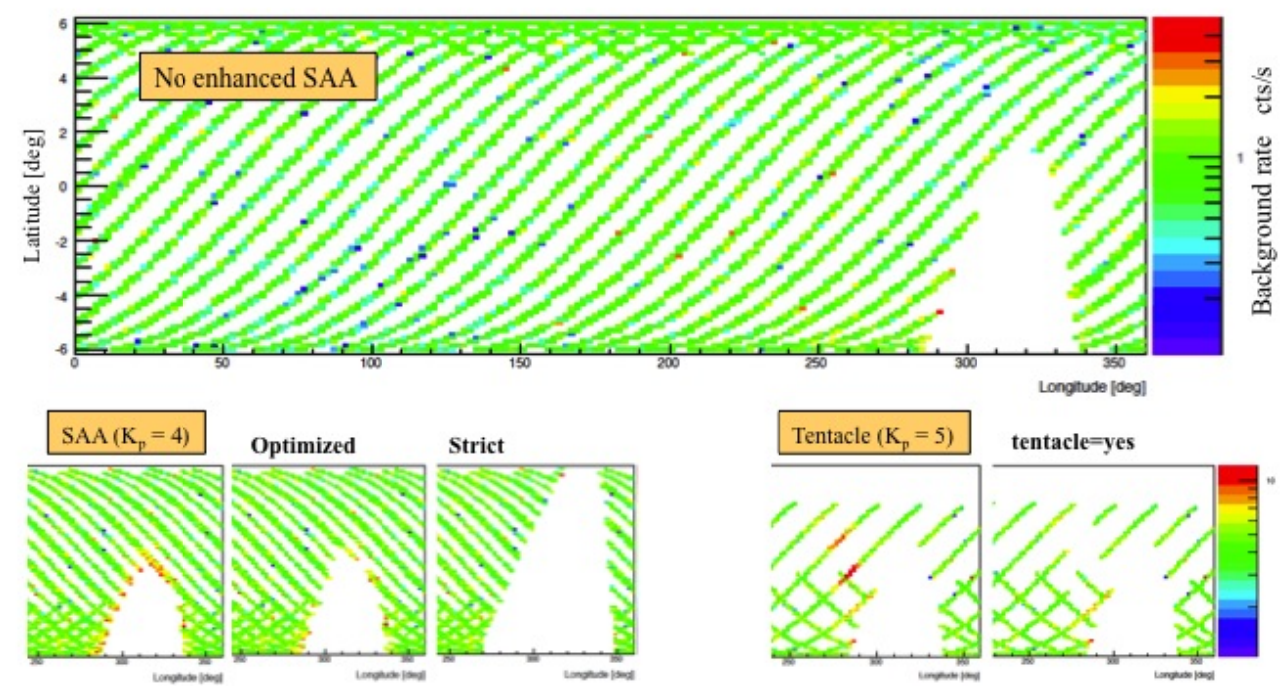

Figure 5. NuSTAR ground trace plots for GTI with the color-coded backgound event rate. The Northern tip of the SAA is the region between longitudes $280^{\circ}<$ Lon $<350^{\circ}$ and latitude $-6^{\circ}<$ Lat $<+2^{\circ}$. Top - Background stability for observation with no major Solar activity. Lower left - Increased detector background when spacecraft is close to the SAA during a $\mathrm{K}_{\mathrm{p}}=$ 4 event, and the effect of available screening options. Lower right - Increased detector background (tentacle) during a minor geomagnetic storm $\left(\mathrm{K}_{\mathrm{p}}=5\right)$ and the effect of screening.

Solar X-ray flares may also be detected as increased background during NuSTAR observations, there is no automated screening in NuSTARDAS for Solar flare events, and users must manually set the GTI for the observation to exclude the time around the event.

\subsection{Mast Thermal Motion}

The mast structure was designed to minimize thermal flexing and residual motions are tracked using the laser metrology system, which provides $0.1^{\prime \prime}$ accuracy to the determined location of the optical axis $(\mathrm{OA})$ on the focal plane. Detailed simulations before launch indicated that the relative motion of the OA of the mirror assembly would be on average $3 \mathrm{~mm}$ $\left(\sim 1^{\prime}\right)$ across the focal plane detectors. The motion of the optical axis is centered on different locations on the detectors that depend on the angle of illumination of the spacecraft by sunlight. This was verified with a series of observations across the sky during performance verification operations. The character and magnitude of the OA motion is almost exactly as modeled before launch.
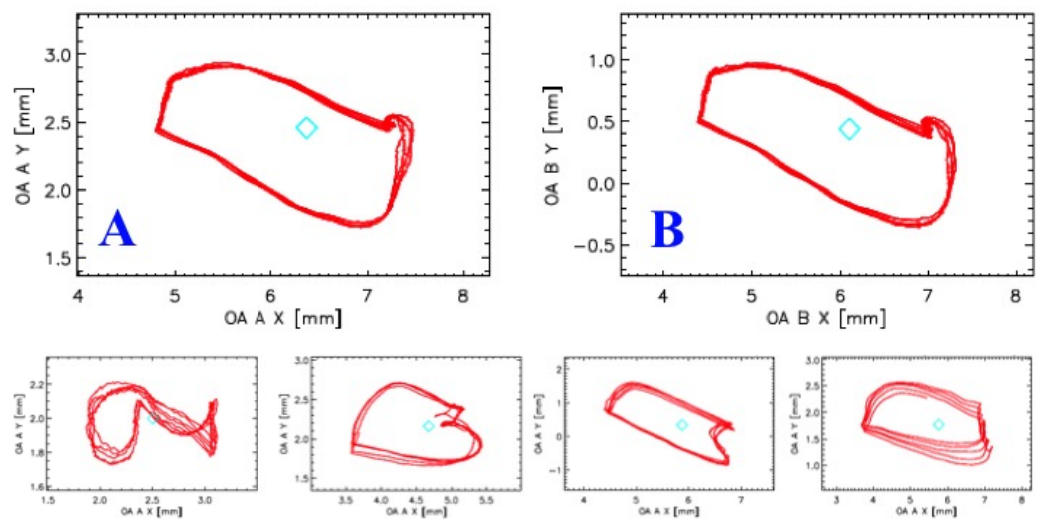

Figure 6. Motion of the optical axis (OA) across the focal plane during an observation. Top: Motion of the OA on FPMA $(A)$ and FPMB $(B)$ for an observation at Solar aspect angle $120^{\circ}$. Lower: Motion of the OA on FPMA for observations at Solar aspect angle $51,83,124,170^{\circ}$ (left to right). 
The sky position of the optical axis as a function of time is determined in NuSTARDAS using the metrology data and this is then used to calculate the time dependent off-axis angle of a given celestial source and hence the effective area correction factor for the observation. The location of the center of motion of the optical axis can be adjusted using the mast adjustment mechanism (see Fig. 1). The optimal location of the OA is away from the gaps between the detectors and within the calibrated range on the laser metrology system was determined during observatory performance verification. This nominal position is located on DET0 in each focal plane assembly (see Fig. 3). For most observations the location of the OA is closer to the detector gaps then the width of the instrument PSF and so most targets are placed a few $\mathrm{mm}$ from the optical axis as a compromise between the loss of photons falling in the detector gaps and the lower offaxis effective area away from the position of the OA.
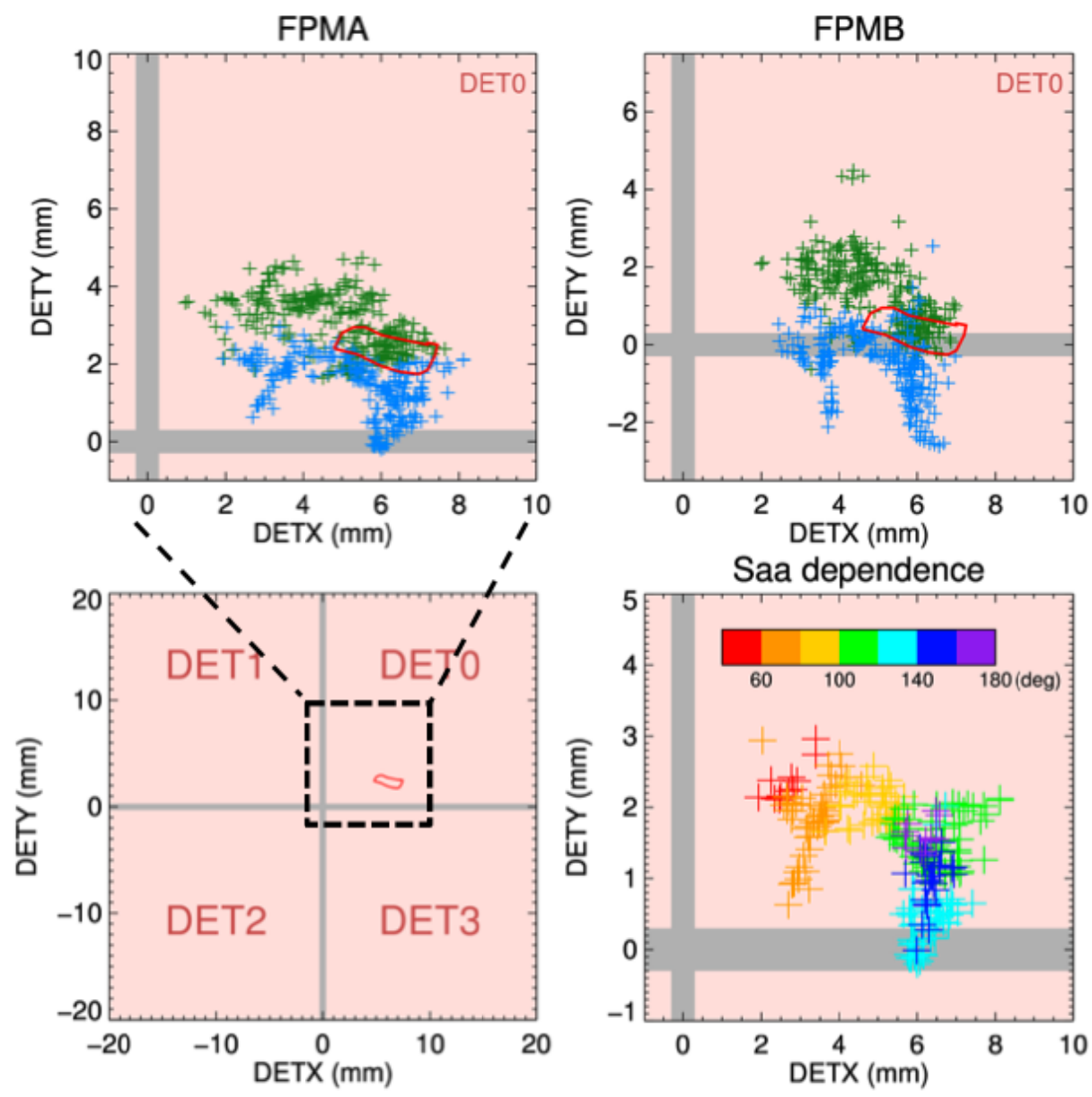

Figure 7. Mean position of the telescope optical axis (OA) motion on the focal plane detectors. Lower-Left - Arrangement of the four CZT detectors in each focal plane module. The $0.6 \mathrm{~mm}$ gaps between the CZT detectors is shown in gray. A typical motion of the OA during an observation is 3mm (red oval on DET0). Top-Left - Centroid of the motion of the OA on FPMA for each observation before (green) and after (blue) an OA position adjustment on 2013-09-17. A sample motion of the OA during one observation is shown in red. The character of the motion changes with differing thermal conditions (see Fig. 6). Top-Right - The OA position on FPMB. The twisting of the mast with differing thermal conditions results in the difference in the OA position between the two telescopes. Lower-Right - FPMA OA position for observations after 2013-09-17. The color-coding represents the average Solar aspect angle during each observation.

\subsection{Attitude pointing stability}

One of the primary challenges of the early mission was managing the changing alignment of the star trackers with pointing direction, which can cause changes in pointing and potentially place targets in sub-optimal locations on the focal plane. There are multiple elements to each calculation of the commanded attitude of the spacecraft that will place the target at the desired location on the focal plane. 
1. Offset between spacecraft $Z$-axis alignment and telescope boresight.

2. Dependence of spacecraft ACS solution with solar illumination of spacecraft.

3. Relative offsets of ACS solution from various combinations of CHU.

4. Expected centroid of OA motion on focal plane and the importance of events with $\mathrm{E}>30 \mathrm{keV}$.

The primary constraint on the spacecraft attitude is the requirement to keep the Sun on the side of the observatory where the solar array is located. Pointing at a celestial target thus involves a rotation of the spacecraft about the Sun-Earth vector with a small margin to keep the Sun within $+2^{\circ}$ to $-10^{\circ}$ normal to the solar array. So for any particular target the position angle for the observation is determined by the date of the observation.

(1) It was recognized early during the performance verification phase that the commanded sky position did not place the target in the field of view of the instrument. The SOC calculates an offset pointing coordinate based on the J2000 coordinates of the target, the position angle for the observation, and the desired location of the target on the focal plane. Offset pointing coordinates are usually within $46^{\prime \prime}-48^{\prime \prime}$ of the J2000 target coordinates and the commanded inertial attitude quaternion is calculated based on these offset coordinates.

(2) The nominal desired location of the target on the focal plane is on the DET0 detector at $[\mathrm{X}, \mathrm{Y}]=[+4.0,+4.0] \mathrm{mm}$, this keeps the target away from the gaps between the detectors and close enough to the expected location of the optical axis. An additional correction to the aim point is determined from inspection of the focal plane locations of targets in recent observations, within $\sim 40$ days and $\sim 50^{\circ}$ of the planned target. The locus of this reference target coordinate on the focal plane is compared to the desired location for that observation, and the aim point for the new observation is adjusted accordingly. The closer the reference target is, in time and sky coordinates, the more successful the correction.

(3) The spacecraft ACS combines the attitude solution of the three CHU on the spacecraft bus and is designed to actively control the attitude to within 1" during an observation, performing adjustments to keep the spacecraft $+\mathrm{Z}$ axis oriented towards the science target. However, the complex Solar illumination and shadowing of the three CHU, and the thermal flexing of the spacecraft components they are mounted to, changes the alignment of each CHU and results in an additional uncertainty in the attitude solution.
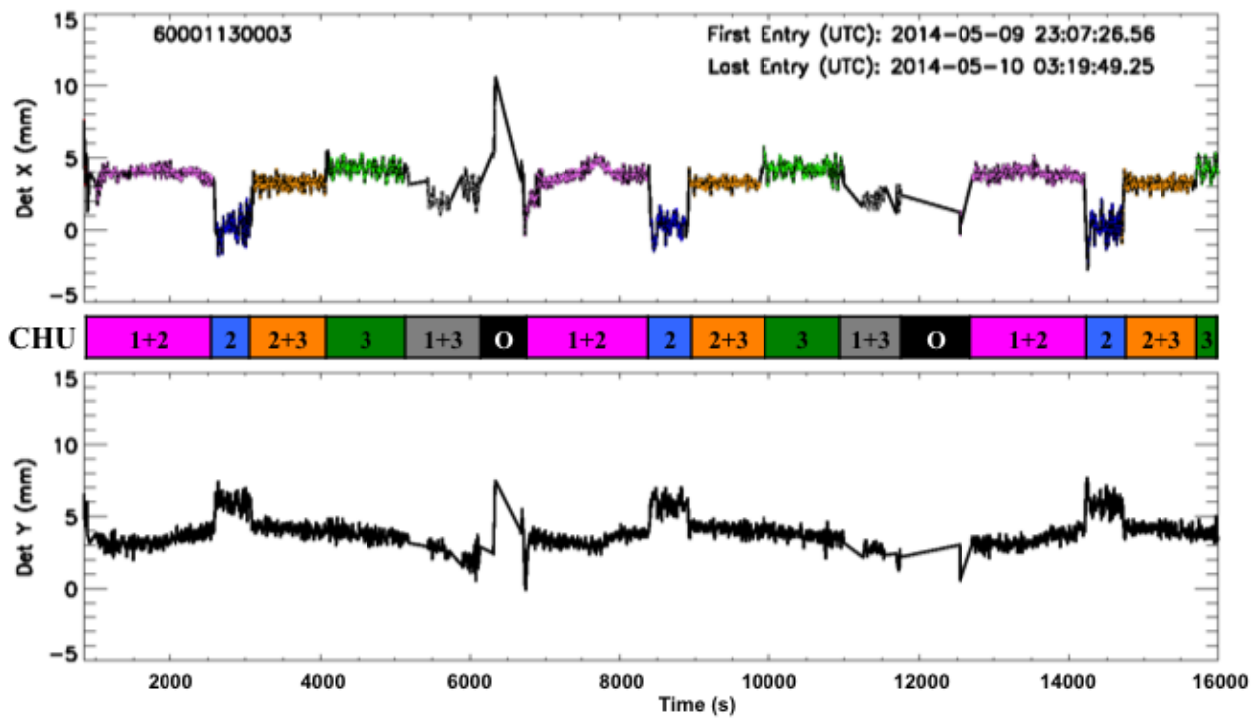

Figure 8. Motion of target coordinates in detector X Y direction for 3 orbits $\left(3 \mathrm{~mm} \sim 1^{\prime}\right)$. No absolute astrometry is available during the short periods when CHU4 is occulted by the Earth $(\mathrm{O})$. The pattern of valid attitude CHU combinations (color coded bar) driving the spacecraft ACS repeats each orbit as CHU1,2,3 become occulted by the Earth.

The complex shadowing and illumination by the Sun of the locations where the CHU are mounted changes the alignment of the CHU in a way not accounted for in the on-board ACS calibration matrices and flight software. The combination of attitude information from the CHUs fed into the spacecraft ACS changes as the CHU field of view become occulted by the Earth during each orbit. Fig. 8 above shows how the combination of various CHU contributing to the ACS changes the absolute astrometry during three orbits of an observation. The ACS will perform small 
adjustments to the spacecraft attitude to keep the spacecraft at the "correct" attitude. This pattern is repeated throughout the observation, only slowly changing as the NuSTAR orbit precesses and the occultation timing for the CHUs change.

The impact of this additional motion on the scientific quality of most observations is minimal because $90 \%$ of the total on-target time, for observations at almost all Solar aspect angle, places targets within $\pm 1.5 \mathrm{~mm}\left(30^{\prime \prime}\right)$ of the centroid of the motion. Fig. 9 shows a contour image of the relative motion of the target position on the focal plane taken from over 800 observations ( $>3 \times 10^{6}$ measurements) during the first two years of the mission. The relative positions are normalized with the centroids for each observation placed at $[6,6]$. The plot has a logarithmic scale with $90 \%$ of all target position measurements within the red contour area.

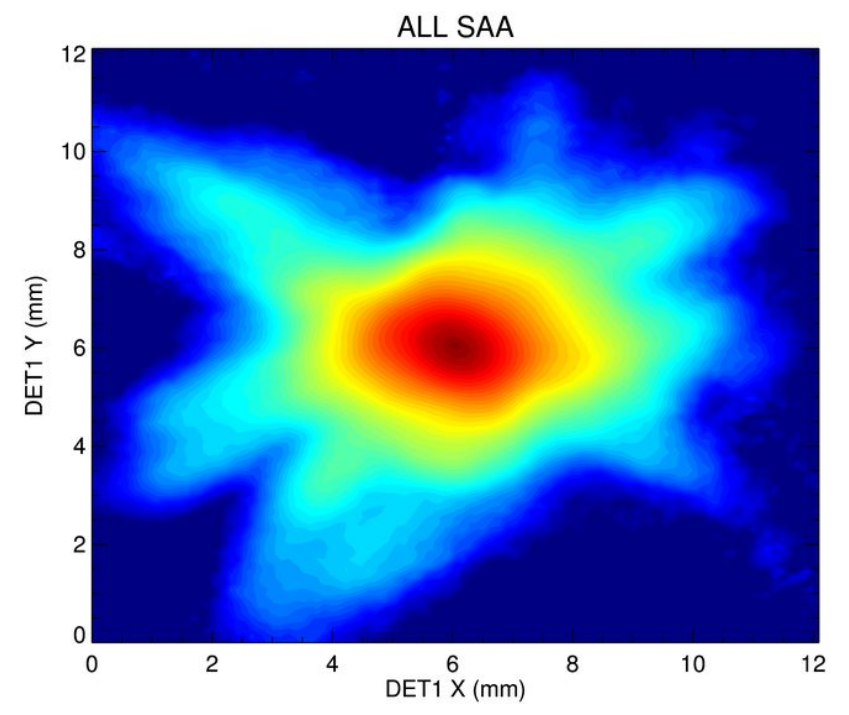

Figure 9. Contour image of target positions on the focal plane normalized to the centroid of the motion in each observation (placed at $[6,6]$ ). The contour has a logarithmic scale to show the extent of the relative motion. $90 \%$ of the total on-target time for the mission has target positions within $\pm 1.5 \mathrm{~mm}$ ( $30^{\prime \prime}$; red area) of the centroid of the motion.

\section{CONCLUSION}

There has been no measureable degradation in the NuSTAR instrument or spacecraft performance since launch, with only expected adjustments to shield threshold levels and nominal OA location performed as the observatory ages onorbit. Operations costs are very low, a consequence of significant automation in mission operations, with science operations performed by a small team of scientists and engineers at the SOC.

The response of the 2014 NASA Senior review to the proposed extension of NuSTAR operations past the primary mission was very positive; NuSTAR was ranked second out of the nine missions reviewed, and NASA has directed the project to implement a Guest Observer (GO) program in 2015. Half of the observing time will be made available to the community in the GO program, split between the NuSTAR cycle 1 (6.5 Msec), XMM-Newton cycle 14 (1.5 Msec) and Chandra cycle $17(0.5 \mathrm{Msec})$ solicitations. The NuSTAR cycle 1 solicitation will also include the opportunity to propose for joint Suzaku $(0.5 \mathrm{Msec})$ observation programs. The importance of 'unplanned' observations has also been recognized by the project and $15 \%$ of observing time has been allocated to $\mathrm{ToO}$ and directors discretionary time.

The ability deploy an X-ray optics on-orbit has enabled a low cost solution for X-ray telescopes and is paving the way for a new generation of space observatories; the next implementation of a similar system will be on the ASTRO-H $\operatorname{mission}^{16}$.

NUSTAR is providing astronomers with image and spectral resolution an order of magnitude higher then previous astrophysics missions operating at hard X-ray energies and is close to completing a two year primary mission. There have been more then 50 papers published in refereed journals so far, making fundamental advances in areas of central importance to astrophysics ${ }^{17}$. The NuSTAR observatory has been highly productive on a small budget and is providing a great return on NASA's investment. 


\section{ACKNOWLEDGEMENTS}

This work was supported under NASA contract No. NNG08FD60C, and made use of data from the NuSTAR mission, a project led by the California Institute of Technology, managed by the Jet Propulsion Laboratory, and funded by the National Aeronautics and Space Administration. We thank the NuSTAR operations, Software and Calibration teams for support with the execution and analysis of these observations. This research has made use of the NuSTAR Data Analysis Software (NuSTARDAS) jointly developed by the ASI Science Data Center (ASDC, Italy) and the California Institute of Technology (USA).

\section{REFERENCES}

[1] Harrison, F.A., Craig, W.W., Cristensen, F.E. et al., "The Nuclear Spectroscopic Telescope Array (NuSTAR) HighEnergy X-ray Mission”, The Astrophysical Journal, 770, 103 (2013).

[2] Jorgensen, P.S., Jorgensen, J.L., and Denver, T., in "Proceedings of the 4S Symposium: Small Satellites, Systems and Services" Ed. B. Warmbein, v571 (ESA SP-571) (2004).

[3] Petre, R. and Serlemitos, P., "Conical Imaging Mirrors for High-Speed X-ray Telescopes", Applied Optics 241833 (1985).

[4] Koglin, J. E. et al., "NuSTAR hard x-ray optics design and performance", Society of Photo-Optical Instrumentation Engineers (SPIE) Conference Series 7437 (2009).

[5] Madsen, K. et al., "Optimizations of $\mathrm{Pt} / \mathrm{SiC}$ and W/Si multilayers for the Nuclear Spectroscopic Telescope Array", Society of Photo-Optical Instrumentation Engineers (SPIE) Conference Series 7437 (2009).

[6] Christensen, F. E. et al., "Coatings for the NuSTAR mission," Society of Photo-Optical Instrumentation Engineers (SPIE) Conference Series 8147 (2011).

[7] Kitaguchi, T. et al., "Inflight performance and calibration of the NuSTAR CdZnTe pixel detectors", Society of Photo-Optical Instrumentation Engineers (SPIE) Conference Series 9144 (2014).

[8] Roberts, B. et al., "Highly automated on-orbit operations of the NuSTAR telescope", Society of Photo-Optical Instrumentation Engineers (SPIE) Conference Series 9149 (2014).

[9] Apache Ubversion, URL: http://subversion.apache.org (2014).

[10]NASA's High Energy Astrophysics Science Archive Research Center, URL http://heasarc.gsfc.nasa.gov (2014).

[11] Pence, W. "NASA office of guest investigator programs (OGIP) FITS format recommendations", URL: http://heasarc.gsfc.nasa.gov/docs/heasarc/ofwg/ofwg_intro.html (2011).

[12] Pence, W. "FITSIO, v2.0: A New Full-Featured Data Interface" in ASP Conf. Ser. Vol. 172. Astronomical Data Analysis Software and Systems VIII, ed. D.Mehringer, R. Plante, and D. Roberts (San Francisco: ASP), 487 (1999).

[13] NASA/IPAC Infrared Science Archive (IRSA). URL: http://irsa.ipac.caltech.edu/frontpage/ (2014).

[14] The Barbara A. Mikulski Archive for Space Telescopes (MAST), URL: https://archive.stsci.edu (2014).

[15] Madsen, K.K. et al. "NuSTAR in-orbit calibration”, The Astrophysical Journal, in preparation (2014).

[16] Takahaski, T. et al., "The ASTRO-H Observatory" in "Space telescopes and Instrumentation 2012: Ultraviolet to Gamma Ray, Proceedings of the SPIE" v8443, 84431A (2012).

[17] Madsen, K.K. et al., "The Nuclear Spectroscopic Telescope Array (NuSTAR) High-energy X-ray Mission”, Society of Photo-Optical Instrumentation Engineers (SPIE) Conference Series 9144 (2014). 